\title{
Developing problem solving competences through the resolution of contextualized problems with an Advanced Computing Environment
}

\author{
Barana, Alice ${ }^{a}$; Fioravera, Michele ${ }^{a}$ and Marchisio, Marina ${ }^{a}$ \\ ${ }^{\mathrm{a}}$ Department of Mathematics, University of Turin, Italy.
}

\begin{abstract}
The paper shows how problem solving competences can be developed by solving contextualized problems using an Advanced Computing Environment (ACE). An ACE is a computer system which enables its user to perform numeric and symbolic computations, graphical representations in two and three dimensions, insert embedded components and create interactive worksheet, all in the same user-friendly environment. An ACE allows students to approach a problematic situation in the way that most suits their thinking, to use different types of representations according to the chosen strategy and to display the whole reasoning together with verbal explanation in the same page: in other words, they can fulfill all the processes that problem solving involves.
\end{abstract}

This paper analyzes a problem solving activity with an ACE proposed by the Department of Mathematics of the University of Turin, and clarifies, also through examples, how the use of the ACE makes it possible to solve real and relevant problems, facilitates the comprehension of the situation and of the Mathematics laying behind and enhance critical thinking.

Keywords: Advanced Computing Environment; Contextualized problems; Critical thinking; Mathematical competence; Mathematical representations; Problem Solving. 


\section{Introduction}

The development of problem solving skills has become a major concern of Mathematics education since it has been chosen as a measure of evaluation of instructional systems (PISA, 2003). European guidelines ask schools to prepare tomorrow's citizens to adapt to ever-changing works and to a world that is more and more dependent on technologies (European Commission, 2015). National educational systems have had to come to terms with this and have issued new indications and actions for teacher training, involving new methodologies rooted in problem posing and solving and in the use of learning technologies (MIUR, 2010) (Brancaccio, et al., 2015). The request of making students able to deal with real problems has recently landed into universities, which traditionally pay less attention to didactic approaches.

The Department of Mathematics of the University of Turin has developed an innovative methodology for the development of problem solving competences, to be proposed to secondary school and university teachers and students, deeply grounded on the use of an Advanced Computing Environment (ACE). ACE is a system which allows to enhance the capabilities of scientific objects representation through numeric computations, symbolic calculus, geometric visualizations in two and three dimensions, and embedding of interactive components while maintaining the ease and immediacy of use of a common word processor. Problem posing and problem solving using an ACE thus require to focus on the chosen problematic situation, which must be real or multidisciplinary, and on the modality in which the ACE is used to find solutions. Purpose of this paper it to show how solving contextualized problems with an ACE can help develop problem solving skills. The discussion is held around examples from a project where these methodologies have been adopted.

\section{State of the art}

While the value of problem solving has been known since Polya's work (Polya, 1957), how the use of technologies affects problem solving activity is a more recent issue. This new theoretical framework is based on the assumption that Mathematical problem solving involves two deeply intertwined processes: the mathematical modellization of the situation and the expression of the reasoning (Carreira, et al., 2016). Modellization cannot take place entirely into the students' mind: real-world and complex situations must be conveyed through a variety of interacting media, which may include words, numbers, symbols, graphics, simulations and many others (Lesh \& Leher, 2009). Representation is thus at the core of relevant problem solving, and the multimodal possibilities of representation offered by different computerized systems enable and enhance different approaches to the problem. 
Mathematics skills not only are needed to find coherent solutions, but are also reinforced after their implementation (Shoenfeld, 1992).

The Department of Mathematics enters this scenery with the proposal of a new methodology based on problem solving activities through an ACE for enhancing learning. It is based on two assumptions: first, problems should be interesting, relevant, open and real situations, even linked to other disciplines; second, an ACE provides all the modalities of representation for one mathematical object, so that students' reasoning can benefit from having a unique environment for solving problems.

\section{3. "Digital Mate Training" to develop problem solving skills}

The activity of problem solving through an ACE has been adopted by the Department of Mathematics in a set of initiatives aimed at enhancing teaching and learning Mathematics. One significant experience has been developed within the project Digital Mate Training (DMT), funded by the Fondazione CRT and carried out through academic years 2014/2015, 2015/2016 and 2016/2017 in collaboration with the ICT Services of the Computer Science Department of University of Turin (Barana, et al., 2016).

\subsection{Description of the project}

The aim of the DMT is to develop and strengthen Mathematics and Computer Science skills through problem solving activities using the ACE Maple and the Virtual Learning Environment (VLE) Moodle. The three editions involved 150 classes of about 40 highschools of Piedmont and Aosta Valley each year, for an amount of 3750 students, 120 teachers and 12 university tutors. After an initial training to solving problems using an ACE that takes place in the laboratories of all the schools, 3 students per class - the most skilled or motivated ones - are enrolled to the VLE and participate to an online training. They are asked to solve a problem every 10 days and to submit their solution, which will be reviewed by the tutors. In the meanwhile, students can participate to weekly synchronous tutoring on the use of Maple and they can collaborate with their colleagues through forum discussions. Students are selected in an intermediate competition and a final one. 


\subsection{Training for the development of competences}

During the online training students learn how to solve problems using the ACE. The problems are graduated in difficulty, so that in the first ones students can focus on the use of the ACE, which is often unknown to them, then complexity progressively increases to make them develop problem solving strategies. The problems content is related to the topics studied at school, so that the participation to the training can really help them get higher understanding of Mathematics. All the problems are based on real-life challenging situations. The texts of the problems are split into smaller questions, all of them are open and increase in complexity. The choice of this structure is due to different reasons: growing difficulty helps students going through the problem without discouraging and it facilitates the assessment, moreover it is similar to the structure of the national final examination of scientific high school, so it helps students practice for it. The final point of all the problems asks to universalize the solution to a more general situation. That can be fulfilled through Maple's embedded components, which can be programmed to perform computations over the data given in input and to return representations and results. This request forces students to go through an abstraction process and to better understand all the solving steps. Moreover, they need to learn the simple programming language standing behind the ACE, which will turn out to be useful in their studying and working future.

A specific rubric has been developed to assess the students' competence in problem solving using an ACE. It includes five indicators: understanding of the problematic solution, development of a solving strategy, accomplishment of the solving process, argumentation and appropriate use of the ACE. All the works submitted during the training are assessed according to the rubric and feedback is provided so that students can acknowledge what to improve. The same assessment criteria are applied in competitions, which consist of the individual resolution of a given problem in two hours time.

\subsection{How an ACE can help develop problem solving competences: a clarifying example}

Various types of representation enable the resolution of a problematic situation. Starting from mental thinking, the solving process can be carried out through several modalities, such as in words, graphically, algebraically, experimentally through computerized simulations. Being able to properly combine those modalities is a crucial aspect in problem solving. Students are free to choose the solving modalities they prefer. This freedom forces them to constantly validate and carefully justify the reasoning: the efficacy of their solving strategy is as important as the clearness of the expression of their solving process. An ACE allows its users to make use of all the representation modalities in a single environment, thus obtaining high levels of clearness and comprehension. 
One of the first evident ACE features is that it helps deal with computations that would be hard to be done "by hand". This has a double effect: students can concentrate on solving the problem rather than on calculating, and the variety of problems that can be proposed to be solved via an ACE is wider. To support these statements, we'll show the problem proposed to the students of the fourth year of Secondary School in the final competition of DMT 2015/2016, entitled "Gears":

Inside an old mechanical toy there is a mechanism which makes a siren sound when it turns on: it consists of two gears rotating one against the other. On each gear, in a point of the external cogwheel, there is an electrode. At the beginning, the two electrodes are in contact; when the gears start rotating, every time the two electrodes make contact the siren emits a sound which simulates a real siren. The toy works when the two gears have the same radius.

The first question of the problem was the following:

Provide a graphical representation of the situation in this case. For reasons of simplicity, you can suppose that the gears are circular shaped and neglect the teeth, that they have unitary radius and that the electrodes complete one rotation in one time unit.

The use of an ACE allows each student to develop their own solution strategies according to their own reasoning. In fact, all the students answered correctly to this first question, while their answers widely varied in the chosen commands - which reflect the mathematical tools used - in the deepening of the solution given and in the ability to express the reasoning in natural language. The variety of methods that an ACE admits for representing mathematical objects allows students to concentrate on the resolution if they master the commands and features at their disposal. In order to display mathematical objects through an ACE it is necessary to provide their formal characteristics: it means that graphical resolutions are usually enough to demonstrate the comprehension of underlying mathematical models. In the example, comprehension involves mathematical prerequisites such as the concepts of circle, point, translation, rotation and so on. In the ACE, a circle can be represented by expressing its equation or by submitting the coordinates of center and radius. Students chose their preferred (or both) method for representing the wheel and the electrodes, correctly adapting their formulas according to the syntax of the requested command, as shown in Figure 1. However, the lacking knowledge on how to use the ACE does not penalize students, since they could just write down the equations and submit a free-drawn sketch of the situation. 

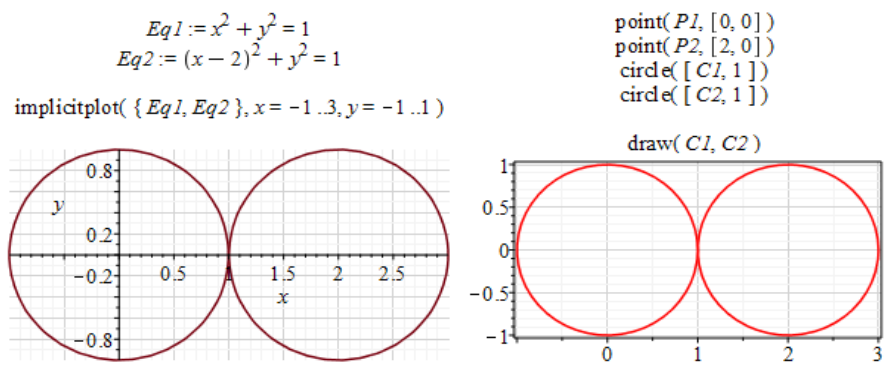

Figure 1. Two different commands entered by students for displaying the wheels.

Well-designed problems often admit a virtually infinite number of different choices for a solution representation, which favors originality in problem solving. In the example, choices widely varied from the essential representation of a couple of trajectories (two circles), to two circles and two points, to the animation of the circles and points. Major advances in the representation of the correct solution coincide with high mastery of mathematical knowledge. For instance, in order to animate the wheels approximated graphics, it is required that the students have understood and decided the variable for expressing the animation, the formulas involved and a proper animation range, as shown in Figure 2 .

$$
\begin{gathered}
\text { animate (pointplot, }[[\cos (2 \pi \cdot t), \sin (2 \pi \cdot t)]], t=0 . .1) \\
\text { animate }(\text { pointplot, }[[\cos (\theta), \sin (\theta)]], \theta=0 . .2 \pi)
\end{gathered}
$$

Figure 2. Two different commands for creating an animated graphic of the electrodes rotating around the wheel elaborated by students. The choice of parametrization for the rotation reveals the different reasoning.

Learning the ACE's programming language helps put into practice the solving modalities. Computational thinking facilitates the students' approach to the problem: they can split the solution into single steps, focus on steps connection, simplify the reasoning by building original procedures and objects. Interactive components can be used to develop two solving modalities within an ACE: generalization and demonstration of particular cases (deductive reasoning), and exploration and recognition of particular cases (inductive reasoning). In some particular cases, inductive reasoning can be useful to understand, but it is not rigorous in Maths and it can lead students to make mistakes. Let's consider the second question:

If the two gears have different radius, how must the radii ratio be in order for the toy to work? Explore this situation through interactive components, then find a relation that the radii ratio must satisfy in order for the mechanism to work.

Students managed to represent the generalization of the problematic situation via a proper and effective development of interactive components. However, many students managed to provide only a particular case of the more general solution, the one they could notice 
through a simulation. The ability of using an ACE cannot overcome mathematical competences: experimental results must be weighted with rigorous calculus. Nevertheless, the use of an ACE can lead student towards correct solutions. In the example, students were close to solving the problem: even if the solutions were not completely correct, they acquired the ability to proceed autonomously through the steps of a resolution of a problem.

\section{Results and discussion}

The results after the DMT proves our thesis that solving contextualized problems through an ACE helps develop problem solving competences. The average score achieved in the final competition in 2015/2016 was 70/100, which is a notably high result. Almost all the participants managed to solve at least one part of the problem assigned, which was more difficult than those assigned during the training. Data about the submissions during the training reveal a lot about the progressive enhancement in problem solving skills of participants. As shown in Figure 3, the average grades achieved by students problem after problem increased, starting from 69 in the first problem and ranging up to 81.5 in the last one, while problems complexity increased as well. All the participants are skilled students in Mathematics: this allowed them to develop problem solving competences, which require the Mathematical knowledge as a background. Self-assessment of the 9 training problems is even more meaningful: students were required to do it after submitting their solutions, and it shows a similar increase. From the evaluation survey distributed at the end of the project to all the participants, it emerged that the use of the ACE and interesting problems were the features of the project which most engaged the students: the use of the ACE was considered engaging with a mean value of 4.0 (st. dev.: 1.0) and the problems with 3.8 (st. dev.: 0.8). Lastly, they considered that solving problems through an ACE was very useful for several reasons, as shown in the Table 1; their answers are compared with those of their teachers.

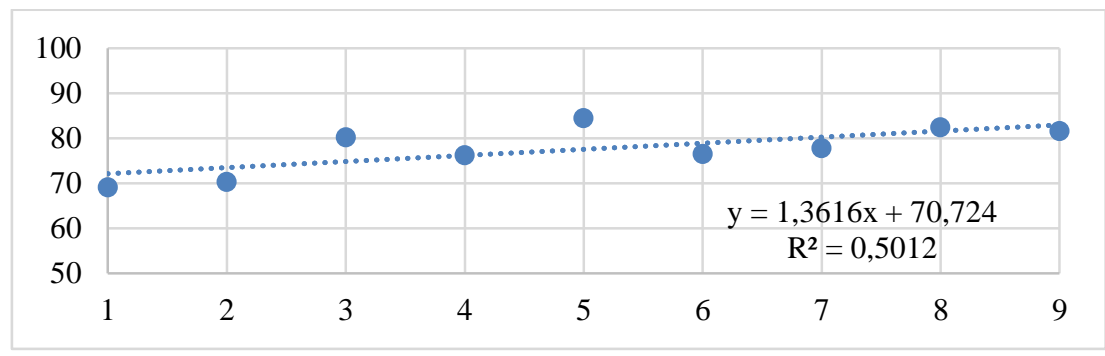

Figure 3. Average students' grades of the 9 training problems 


\section{Conclusions}

The experience of the DMT project shows that solving contextualized problems through an ACE is a useful activity for the development of cross-cutting competences, first of all problem solving. It is important that Mathematics teachers include these kinds of activities in their classes: the teachers of the classes participating to the DMT receive a special training aimed at adopting these methodologies into their teaching. As a result, many of them started using problem solving activities with the ACE in their classes, and several of their students who didn't participate to DMT asked Maple's licenses on their computers.

The Department is particularly dedicated to this purpose and includes this methodology in several other projects aimed at teacher training. The major difficulty for teachers is their inexperience in the creation of cross-disciplinary problems: one possible solution could be to organize multidisciplinary activities or courses, with the collaboration of teachers and experts of different subjects, with the special purpose of developing problem solving skills. The new "Alternanza Scuola Lavoro", now compulsory for all Italian high schools (MIUR, 2015), provides a first answer to this request; the DMT project, for the reasons discussed above, has been proposed as an activity of Alternanza Scuola Lavoro (Barana \& Marchisio, 2016).

Table 1. Usefulness of the problem solving with an ACE according to students and teachers.

\begin{tabular}{ccccc}
\hline & \multicolumn{2}{c}{ Students } & \multicolumn{2}{c}{ Teachers } \\
\hline & Average & St. Dev. & Average & St. Dev \\
\hline Better understanding of some aspects of Mathematics & 3.3 & 1.1 & 3.9 & 0.8 \\
Deepen links between Mathematics and Informatics & 3.7 & 1.1 & 4.1 & 0.7 \\
Better understanding of the usefulness of Math & 3.6 & 1.1 & 4.0 & 0.7 \\
Acquire useful skills for school and university & 3.9 & 0.9 & 4.3 & 0.7 \\
Acquire skills spendable in the working world & 3.7 & 1.1 & 4.0 & 0.9 \\
\hline
\end{tabular}

\section{Acknowledgment}

The authors desire to thank the Fondazione CRT of Turin, in particular dott.ssa Daniela Tornielli for the support given for the Project Digital Mate Training and for her remarkable dedication and passion. 


\section{References}

Barana, A., \& Marchisio, M. (2016). Dall'esperienza di Digital Mate Training all'Alternanza Scuola Lavoro. Atti di Didamatica. Udine.

Barana, A., Marchisio, M., \& Rabellino, S. (2016). Assessment of individual and collaborative e-learning in problem solving activities. Proceedings of EMEM Italia.

Brancaccio, A., Demartini, C., Marchisio, M., Palumbo, C., Pardini, C., Patrucco, A., \& Zich, R. (2015). Problem Posing and Solving: Strategic Italian Key Action to Enhance Teaching and Learning of Mathematics and Informatics in High School. Proceedings of COMPSAC Symposium on Computer Education and Learning Technologies (CELT).

Carreira, S., Jones, K., Amando, N., Jacinto, H., \& Nobre, S. (2016). Youngsters Solving Mathematical Problems with Technology. Springer.

European Commission. (2015, September 25). EUROPE 2020 - A strategy for smart, sustainable and inclusive growth. Europe 2020: an Overview, Annex 1.

Lesh, R., \& Leher, R. (2009). Models and Modeling Perspectives on the Development of Students and Teachers. Mathematical Thinking and Learning, 5(2\&3), 109-129.

MIUR. (2010). Schema di regolamento recante "Indicazioni nazionali riguardanti gli obiettivi specifici di apprendimento concernenti le attività e gli insegnamenti compresi nei piani degli studi previsti per i percorsi liceali". Roma.

PISA. (2003). Literacy Skills for the World of Tomorrow. OECD/UNESCO.

Polya, G. (1957). How to Solve It. Princeton University Press.

Shoenfeld, A. H. (1992). Learning to think mathematically. In D. A. Grouws, Handbook of research on mathematics teaching and learning (334-370). New York: Macmillan 